\title{
Transverse Coronal-Loop Oscillations Induced by the Non-radial Eruption of a Magnetic Flux Rope
}

\author{
Q.M. Zhang ${ }^{1,2}$ (D) J.L. Chen ${ }^{1,2}$ (D) \\ S.T. $\mathrm{Li}^{1,2} \cdot$ L. $\mathrm{Lu}^{1,2}$ (D) D. $\mathrm{Li}^{1,2}$ (D)

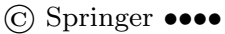

\begin{abstract}
We investigate the transverse coronal-loop oscillations induced by the eruption of a prominence-carrying flux rope on 7 December 2012. The flux rope originating from NOAA Active Region (AR) 11621 was observed in extremeultraviolet (EUV) wavelengths by the Atmospheric Imaging Assembly (AIA) onboard the Solar Dynamics Observatory (SDO) spacecraft and in $\mathrm{H} \alpha$ line center by the ground-based telescope at the Big Bear Solar Observatory (BBSO). The early evolution of the flux rope is divided into two steps: a slow rise phase at a speed of $\approx 230 \mathrm{~km} \mathrm{~s}^{-1}$ and a fast rise phase at a speed of $\approx 706 \mathrm{~km} \mathrm{~s}^{-1}$. The eruption generates a C5.8 flare and the onset of the fast rise is consistent with the hard X-ray (HXR) peak time of the flare. The embedded prominence has a lower speed of $\approx 452 \mathrm{~km} \mathrm{~s}^{-1}$. The eruption is significantly inclined from the local solar normal by $\approx 60^{\circ}$, suggesting a typical non-radial eruption. During the early eruption of the flux rope, the nearby coronal loops are disturbed and experience independent kink-mode oscillations in the horizontal and vertical directions. The oscillation in the horizontal direction has an initial amplitude of $\approx 3.1 \mathrm{Mm}$, a period of $\approx 294$ seconds, and a damping time of $\approx 645$ seconds. It is most striking in $171 \AA$ and lasts for three to four cycles. The oscillations in the vertical directions are observed mainly in 171,193 , and $211 \AA$. The initial amplitudes lie in the range of $3.4-5.2 \mathrm{Mm}$, with an average value of $4.5 \mathrm{Mm}$. The periods are between 407 seconds and 441 seconds, with an average value of 423 seconds. The oscillations are damping and last for nearly four cycles. The damping times lie in the range of $570-1012$ seconds, with an average value of 741 seconds. Assuming a semi-circular shape of the vertically oscillating loops, we calculate the loop lengths according to their heights. Using the observed periods, we carry out coronal seismology and estimate the internal Alfvén speeds
\end{abstract}

\footnotetext{
$凶$ Q.M. Zhang

zhangqm@pmo.ac.cn

1 Key Laboratory of Dark Matter and Space Astronomy, Purple Mountain Observatory, CAS, Nanjing 210023, China

2 School of Astronomy and Space Science, University of Science and Technology of China, Hefei 230026, China
} 
$\left(988-1145 \mathrm{~km} \mathrm{~s}^{-1}\right)$ and the magnetic-field strengths $(12-43 \mathrm{G})$ of the oscillating loops.

Keywords: Prominences, Active; Coronal Mass Ejections, Initiation and Propagation; Magnetic fields, Corona; Waves, Magnetohydrodynamic

\section{Introduction}

A magnetic flux rope is a bundle of twisted field lines that wind around their common axis (see Liu, 2020, and references therein). The accumulated twist number $\left[\mathcal{T}_{w}\right]$ can reach up to one to three turns (Liu et al., 2016; Guo et al., 2021a). Flux ropes play an essential role in the genesis of solar eruptions (Chen, 2017; Cheng, Guo, and Ding, 2017), including prominence eruptions (Rust and Kumar, 1996; Amari, Canou, and Aly, 2014), flares (Titov and Démoulin, 1999; Janvier, Aulanier, and Démoulin, 2015; Wang et al., 2015), and coronal mass ejections (CMEs: Dere et al., 1999; Vourlidas et al., 2013; Patsourakos et al., 2020). It is still controversial whether flux ropes are generated before eruptions (Canou et al., 2009; Green and Kliem, 2009; Zhang, Cheng, and Ding, 2012; Zhang, Su, and Ji, 2017; James et al., 2018; Yan et al., 2018; Chen et al., 2019; He et al., 2020; Nindos et al., 2020) or during eruptions (Cheng et al., 2011; Gou et al., 2019). Photospheric flux cancellation is found to be important in the formation of flux ropes before eruptions (Green, Kliem, and Wallace, 2011; Savcheva et al., 2012), while tether-cutting magnetic reconnection in the corona is believed to be an effective mechanism of flux-rope formation during eruptions (Joshi, Magara, and Inoue, 2014; Xue et al., 2017). Flux ropes are frequently observed to have very high temperatures $(\approx 10 \mathrm{MK})$, which are best revealed in extreme-ultraviolet (EUV) $94 \AA$ and $131 \AA$ and therefore termed "hot channel" (Cheng et al., 2011, 2012, 2013, 2014). Nindos et al. (2015) analyzed 141 M-class and X-class flares. About $32 \%$ of the events are associated with hot channels and almost half of the eruptive events are related to a hot channel configuration.

The directions of prominence eruptions and associated CMEs are not always radial. McCauley et al. (2015) investigated the properties of 904 prominence and filament eruptions observed by the Solar Dynamics Observatory (SDO) in detail. It is found that the percentage of non-radial eruptions reaches $12 \%$. Devi et al. (2021) reported a non-radial prominence eruption away from the local vertical with an inclination angle of $\gamma=48^{\circ}$, which is attributed to the easier channel provided by the open and high-lying magnetic field. Using stereoscopic observations from the twin spacecraft of the Solar TErrestrial RElations Observatory (STEREO: Kaiser et al., 2008), Gosain et al. (2009) observed a partial filament eruption that was highly inclined to the solar normal with an inclination angle of $\gamma=47^{\circ}$, which is close to that reported by Williams et al. (2005). Combining the observations from the STEREO-Ahead (hereafter STA) and the Atmospheric Imaging Assembly (AIA: Lemen et al., 2012) onboard SDO, Sun et al. (2012a) reported a non-radial, jet-like eruption following a markedly inclined trajectory with $\gamma=66^{\circ}$. Combining the observations from STEREO-Behind (hereafter STB) and SDO/AIA, Bi et al. (2013) investigated the rotation and non-radial 
propagation of a filament, the later of which resulted from interaction between the filament eruption and the overlying pseudo-streamer. In an extreme case, a nearly $90^{\circ}$ deflected filament eruption and the related CME were noticed by Yang et al. (2018). State-of-the-art numerical simulations indicate that the imbalance of the bipole leads to a negative magnetic pressure gradient in the $x$ direction, which prevents the flux rope from expanding symmetrically (Aulanier et al., 2010; Kliem et al., 2013; Inoue et al., 2018; Jiang et al., 2018). Guo et al. (2021b) performed a magnetohydrodynamic (MHD) simulation of a C7.7 class flare, which was generated by a non-radial prominence eruption on 21 June 2011 (Zhou et al., 2017).

Solar eruptions can potentially generate kink-mode, transverse oscillations of the adjacent coronal loops (Aschwanden et al., 1999; Nakariakov et al., 1999; Zimovets and Nakariakov, 2015) and quasi-periodic pulsations (QPP: Zimovets et al., 2021). The polarization of the transverse loop oscillations could be horizontal (White and Verwichte, 2012; Nisticò, Nakariakov, and Verwichte, 2013; Li et al., 2017, 2018; Zhang et al., 2015, 2020; Zhang, 2020; Dai et al., 2021) or vertical (Wang and Solanki, 2004; Gosain, 2012; White, Verwichte, and Foullon, 2012; Simões et al., 2013; Srivastava and Goossens, 2013; Kim, Nakariakov, and Cho, 2014; Dudík et al., 2016; Verwichte and Kohutova, 2017; Reeves et al., 2020). In most cases, the amplitudes of kink oscillations decay with time as a result of resonant absorption, phase mixing, wave leakage, or Kelvin-Helmholtz instability (Goossens, Andries, and Aschwanden, 2002; Ofman and Aschwanden, 2002; Ruderman and Roberts, 2002; Terradas, Oliver, and Ballester, 2006; Goddard et al., 2016; Antolin et al., 2017; Nechaeva et al., 2019). The damping time $[\tau]$ is roughly proportional to the period $[P]$ (Verwichte et al., 2013), and the quality factor $\left[\frac{\tau}{P}\right]$ has a power-law dependence on the amplitude with the exponent of -0.5 (Goddard and Nakariakov, 2016). One of the applications of coronal seismology is the estimation of the coronal magnetic field and Alfvén speed of the oscillating loops (Nakariakov and Ofman, 2001; Aschwanden et al., 2002; Verwichte et al., 2009; Chen and Peter, 2015). So far, there are few reports of transverse coronal-loop oscillations triggered by non-radial prominence eruptions.

On 7 December 2012, a prominence-carrying flux rope erupted from NOAA Active Region (AR) 11621 (N15W91) and propagated non-radially, producing a C5.8 flare and a fast CME. Based on the revised cone model, Zhang (2021) performed a $3 \mathrm{D}$ reconstruction of the CME simultaneously observed by SDO/AIA and STA/EUVI at 21:20:30 UT. The geometry and kinematics of the CME were derived. In this article, we report the simultaneous coronal-loop oscillations in the horizontal and vertical directions induced by the eruption in the same AR. Data analysis is described in Section 2. The results are presented in Section 3 and compared with previous findings in Section 4. Finally, a brief summary is given in Section 5 .

\section{Data Analysis}

The prominence eruption was tracked by the ground-based telescope at the Big Bear Solar Observatory (BBSO) in the $\mathrm{H} \alpha$ line center. The eruption of the flux 
Table 1. Description of the observational parameters.

\begin{tabular}{lcccc}
\hline Instrument & $\begin{array}{c}\lambda \\
{[\AA]}\end{array}$ & $\begin{array}{c}\text { Time } \\
{[\mathrm{UT}]}\end{array}$ & $\begin{array}{c}\text { Cadence } \\
{[\mathrm{s}]}\end{array}$ & $\begin{array}{c}\text { Pixel Size } \\
{\left[{ }^{\prime \prime}\right]}\end{array}$ \\
\hline AIA & $131-211$ & $21: 10-21: 50$ & 12 & 0.6 \\
HMI & 6173 & $21: 10-21: 50$ & 45 & 0.6 \\
EUVI & 195 & $21: 05-23: 05$ & 300 & 1.6 \\
COR2 & WL & $22: 24-23: 39$ & 900 & 14.7 \\
LASCO/C2 & WL & $21: 36-22: 36$ & 720 & 11.4 \\
BBSO & 6563 & $21: 00-21: 50$ & 60 & 1.1 \\
GOES & $0.5-4$ & $21: 00-22: 30$ & 2.05 & $\ldots$ \\
GOES & $1-8$ & $21: 00-22: 30$ & 2.05 & $\ldots$ \\
GBM & $4-26 \mathrm{keV}$ & $21: 05-21: 30$ & 0.256 & $\ldots$ \\
\hline
\end{tabular}

rope was detected by SDO/AIA in EUV wavelengths (131, 171, 193, and $211 \AA$ ). The line-of-sight (LOS) magnetograms of the photosphere were observed by the Helioseismic and Magnetic Imager (HMI: Scherrer et al., 2012) onboard SDO. The AIA and HMI level_1 data were calibrated using the standard Solar Software (SSW) program aia_prep.pro and hmi_prep.pro, respectively. The $\mathrm{H} \alpha$ and EUV images were coaligned using the cross-correlation method.

The eruption was also captured by the Extreme-Ultraviolet Imager (EUVI) in the Sun Earth Connection Coronal and Heliospheric Investigation (SECCHI: Howard et al., 2008) package onboard STA, which had a separation angle of $\approx 128^{\circ}$ with respect to the Sun-Earth direction on 7 December 2012. The CME driven by the flux-rope eruption was observed by the C2 white light (WL) coronagraph of the Large Angle Spectroscopic Coronalgraph (LASCO: Brueckner et al., 1995) onboard the Solar and Heliospheric Observatory (SOHO). The $\mathrm{LASCO} / \mathrm{C} 2$ data were calibrated using the SSW program c2_calibrate.pro. The CME was observed by the COR2 coronagraph onboard STA as well. Calibrations of the COR2 and EUVI data were performed using the SSW program secchi_prep.pro. The deviation of STA north-south direction from the solar rotation axis was corrected. The soft X-ray (SXR) fluxes of the C5.8 flare were recorded by the Geostationary Operational Environmental Satellite (GOES) spacecraft. The hard X-ray (HXR) fluxes at different energy bands were obtained from the the Gamma-ray Burst Monitor (GBM: Meegan et al., 2009) onboard the Fermi spacecraft. The observational properties of the instruments are listed in Table 1.

\section{Results}

\subsection{Eruption of the Prominence-carrying Magnetic Flux Rope}

In Figure 1, the upper panel shows the SXR light curves of the C5.8 flare in 1-8 $\AA$ (red line) and $0.5-4 \AA$ (blue line). It is clear that the SXR flux increases rapidly from $\approx 21: 13: 00$ UT and reaches the peak value at $\approx 21: 21: 15$ UT (black-dashed line) before declining gradually to the pre-flare level around 22:13:00 UT. Hence, 


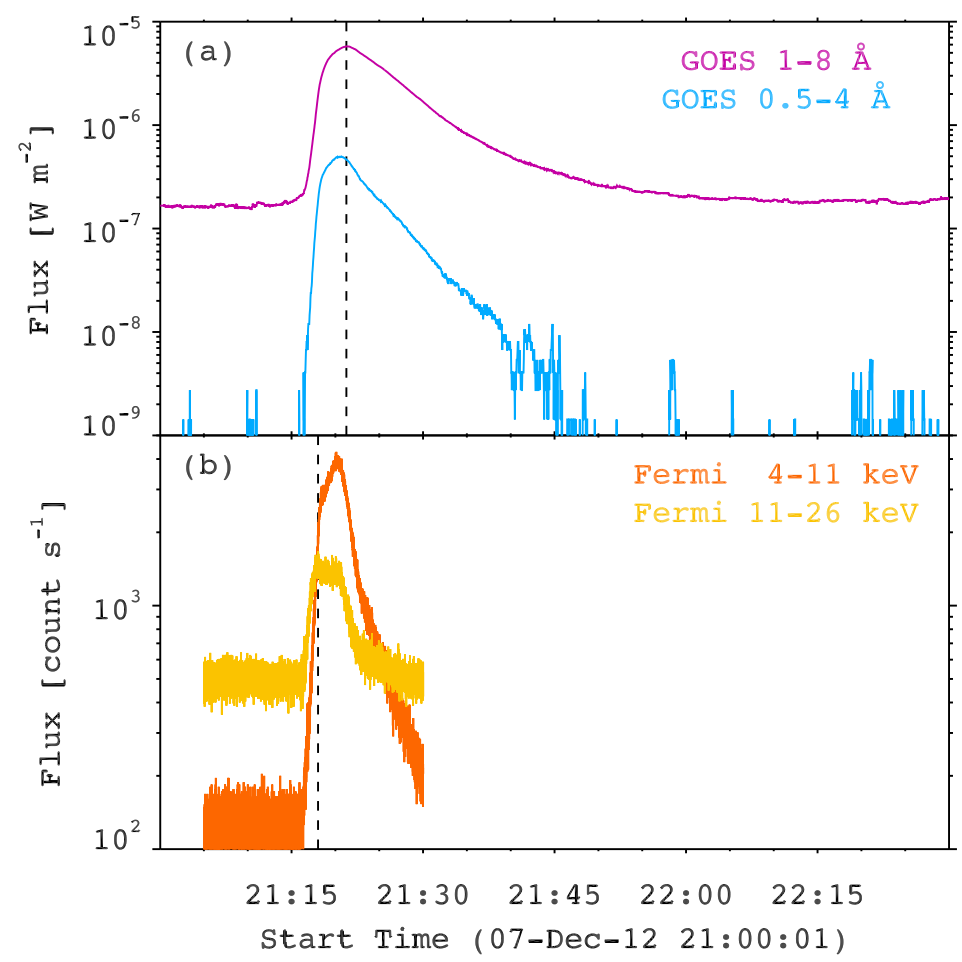

Figure 1. (a) Soft X-ray (SXR) light curves of the GOES C5.8 flare in 1-8 $\AA$ (red line) and 0.5-4 $\AA$ (blue line). The black-dashed line denotes the peak time at 21:21:15 UT. (b) Hard X-ray (HXR) light curves of the flare observed by Fermi/GBM at $4-11 \mathrm{keV}$ (orange line) and $11-26 \mathrm{keV}$ (yellow line). The black-dashed line denotes the HXR peak time at 21:18:00 UT.

the lifetime of the flare is $\approx 1 \mathrm{hr}$. Figure $1 \mathrm{~b}$ shows the HXR light curves at $4-$ $11 \mathrm{keV}$ (orange line) and 11-26 keV (yellow line). The black-dashed line denotes the HXR peak time at $\approx 21: 18: 00$ UT when the rate of energy precipitation of flare-accelerated nonthermal electrons is maximum (Brown, 1971).

Four selected LOS magetograms from 4 December to 7 December 2012 are displayed in Figure 2. It is seen that AR 11621 was still distinguishable on 5 December. As time goes by, it got blurred on 6 December close to the western limb and totally vanished on 7 December, implying that it had rotated to the backside of the Sun when the flare took place.

The EUV images in Figure 3 illustrate the evolution of the prominencecarrying flux rope (see also the Electronic Supplementary Material animaia.mp4). As the flare occurs, the flux rope shows up before 21:18:00 UT (a2-d2). The bubble-like flux rope expands in size and propagates in the southwest direction. An embedded prominence follows the flux rope. It is obvious that the trajectory is severely inclined to the solar normal with $\gamma=60^{\circ}$, meaning that the event is a typical non-radial eruption (Zhang, 2021). The thin leading edge of the flux rope could not be tracked after escaping the field-of-view (FOV) of AIA around 21:21:40 UT. The eruption is evident in various EUV wavelengths, suggesting 


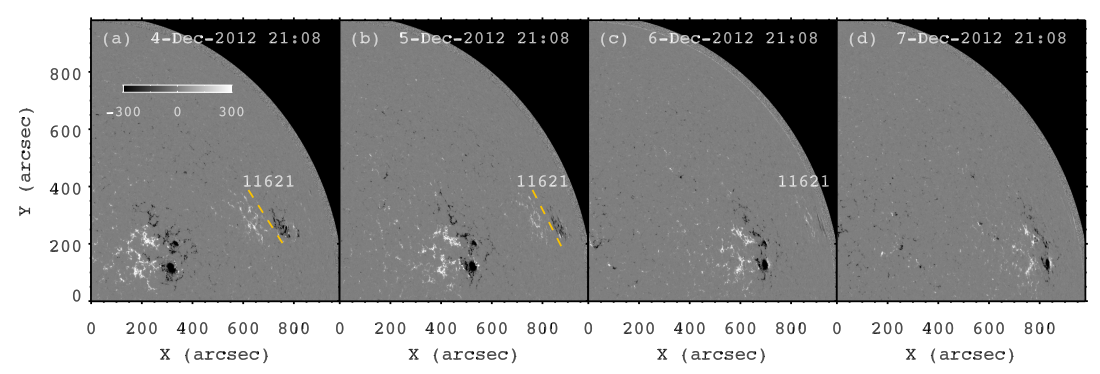

Figure 2. HMI LOS magetograms from 4 December to 7 December 2012. AR 11621 hosting the C5.8 flare is marked. The gold-dashed lines denote the approximate polarity inversion line (PIL).

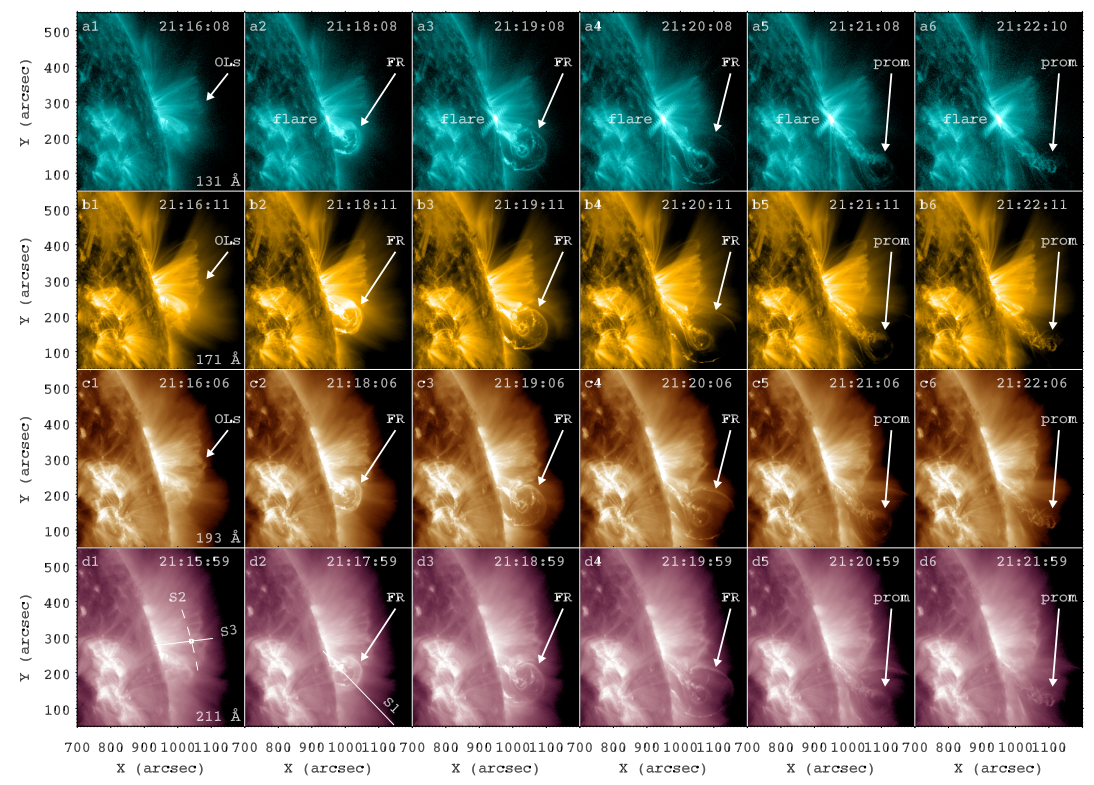

Figure 3. EUV images in $131 \AA$ (a1-a6), $171 \AA$ (b1-b6), $193 \AA(\mathbf{c 1}-\mathbf{c 6})$, and $211 \AA$ $(\mathbf{d} \mathbf{1}-\mathbf{d} \mathbf{6})$. The arrows point to the oscillating loops (OLs), flux rope (FR), and eruptive prominence (prom). In Panel d1, two short slices (S2 and S3) are used to investigate the transverse oscillations of the coronal loops in the horizontal and vertical directions, respectively. In Panel d2, a long slice (S1) is used to investigate the evolution of the flux rope. An animation of this figure is available in the Electronic Supplementary Material (animaia.mp4).

the multi-thermal nature of flux rope and prominence (Hannah and Kontar, 2013). We note that a group of coronal loops to the North of the flux rope are slightly disturbed during the eruption and oscillate for a long time, which will be described in Section 3.2.

The prominence eruption is also vivid in $\mathrm{H} \alpha$ line center (see animation in the Electronic Supplementary Material animha.mp4). Figure 4 shows the $\mathrm{H} \alpha$ images observed by BBSO. The prominence arises slowly at $\approx 21: 16: 00 \mathrm{UT}$ and expands quickly, resembling a tennis racket (see Panel d). The top segment is fitted 


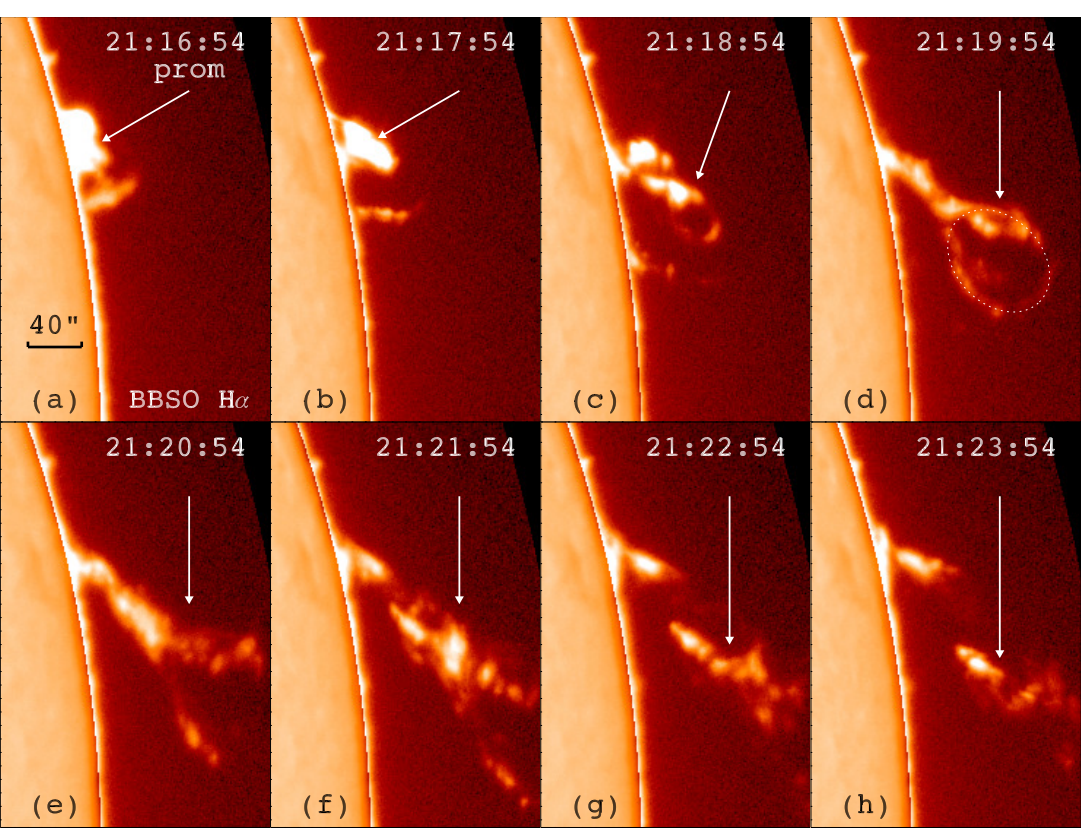

Figure 4. $\mathrm{H} \alpha$ images observed by BBSO. The arrows point to the eruptive prominence (prom). In Panel d, a dotted ellipse is used to fit the top segment of prominence. An animation of this figure is available in the Electronic Supplementary Material (animha.mp4).

with an ellipse (dotted line), whose major axis and minor axis have lengths of $86^{\prime \prime}$ and $64^{\prime \prime}$, respectively. The location and shape of the prominence are consistent with that observed in EUV wavelengths (see Figure 3a4-d4) and are in agreement with the typical U-shaped prominence horns both in observation (Régnier, Walsh, and Alexander, 2011) and numerical simulations (Xia et al., 2014). The prominence continues ascending and the top segment escapes the FOV of BBSO. It is noticed that the bubble-like flux rope in EUV wavelengths is not distinct in $\mathrm{H} \alpha$.

To investigate the evolution of flux rope and prominence, we select a slice (S1) with a total length of $\approx 296^{\prime \prime}$ along the direction of their propagation, which is shown in Figure 3d2. The time-distance diagrams of S1 in 131, 171, 193, and $211 \AA$ are displayed in Figure 5. In Figure 5a, the positions of the flux rope leading edge are labeled with a magenta dashed line, whose slope represents its apparent linear speed $\left(\approx 706 \mathrm{~km} \mathrm{~s}^{-1}\right)$. The positions of the following prominence are labeled with red-dashed lines, whose slopes represent its apparent linear speed $\left(\approx 452 \mathrm{~km} \mathrm{~s}^{-1}\right)$. Hence, the speed of the flux rope is $\approx 1.5$ times larger than the prominence.

As mentioned in Section 1, the eruption of the flux rope drives a fast and wide CME. In Figure 6, the upper and lower panels show the WL images of the CME observed by LASCO/C2 and STA/COR2, respectively. The leading fronts of the CME are pointed to by the arrows. The CME first appears at $\approx 21: 36: 00$ $\mathrm{UT}$ in the $\mathrm{LASCO} / \mathrm{C} 2 \mathrm{FOV}$ and propagates westward with a central position angle of $299^{\circ}$. The CME first appears at $\approx 22: 24: 00 \mathrm{UT}$ in the STA/COR2 FOV 


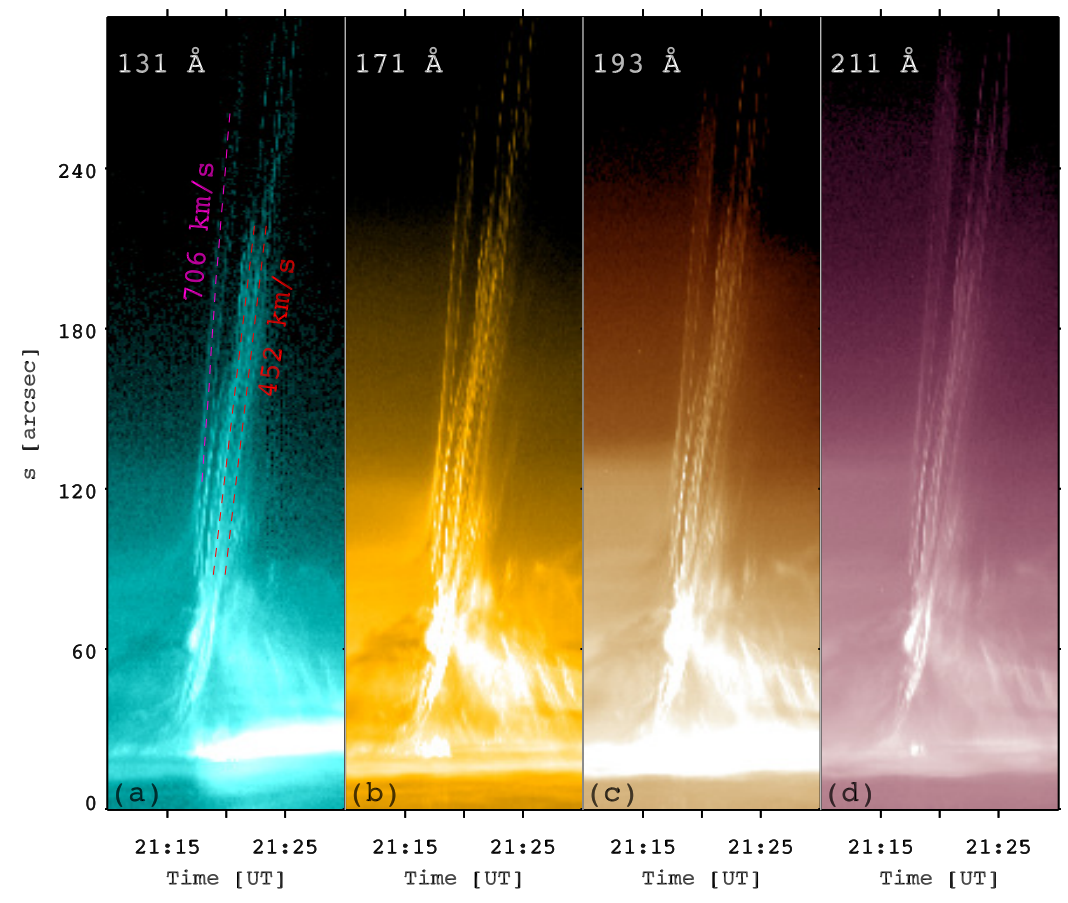

Figure 5. Time-distance diagrams of $\mathrm{S} 1$ in $131,171,193$, and $211 \AA$. The linear velocities of flux rope $\left(\approx 706 \mathrm{~km} \mathrm{~s}^{-1}\right)$ and prominence $\left(\approx 452 \mathrm{~km} \mathrm{~s}^{-1}\right)$ are indicated.

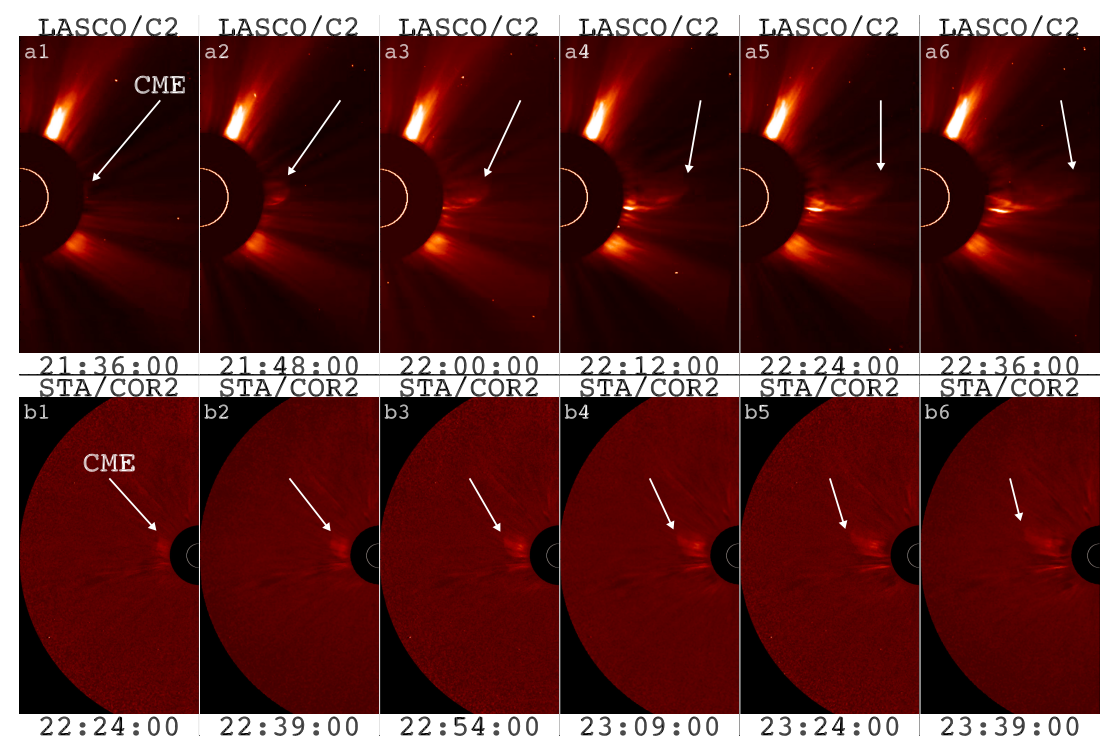

Figure 6. WL images of the CME observed by LASCO/C2 (upper panels) and STA/COR2 (lower panels). The arrows point to the CME leading front. 

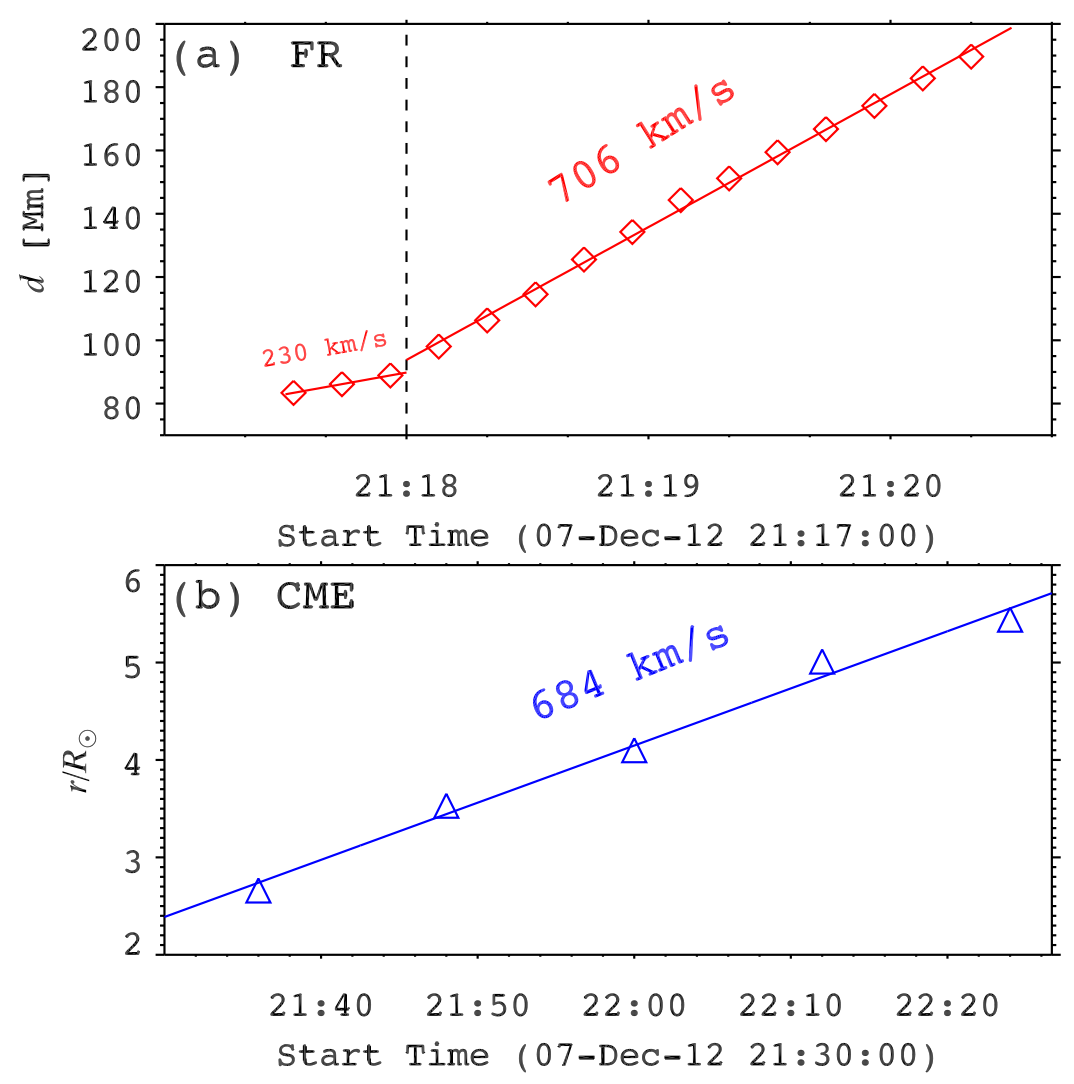

Figure 7. (a) Height variation of the flux-rope leading edge along S1 in $131 \AA$. The apparent linear speeds of slow rise $\left(\approx 230 \mathrm{~km} \mathrm{~s}^{-1}\right)$ and fast rise $\left(\approx 706 \mathrm{~km} \mathrm{~s}^{-1}\right)$ are indicated. The black-dashed line denotes the HXR peak time at 21:18:00 UT. (b) Height variation of the CME leading front observed by LASCO/C2. The plane-of-sky linear speed $\left(\approx 684 \mathrm{~km} \mathrm{~s}^{-1}\right)$ is indicated.

and propagates eastward until $\approx 23: 54: 00 \mathrm{UT}$, when the CME becomes too weak to be identified. It is shown that although the flux rope propagates non-radially in the FOV of AIA, the position angle of the related CME is close to the latitude of AR 11621, implying that the flux rope is probably influenced and redirected by the large-scale magnetic field.

In Figure 7, the upper panel shows the height evolution of the flux rope leading edge in $131 \AA$, which is determined manually in the direction of S1. The movement of the flux rope is roughly divided into two phases by the HXR peak (black-dashed line): a slow rise $\left(\approx 230 \mathrm{~km} \mathrm{~s}^{-1}\right)$ and a fast rise $\left(\approx 706 \mathrm{~km} \mathrm{~s}^{-1}\right)$, which is in line with previous observations (Cheng et al., 2013). The height evolution of the CME leading front in the $\mathrm{FOV}$ of $\mathrm{LASCO} / \mathrm{C} 2$ is plotted in the lower panel of Figure 7 . The apparent speed $\left(\approx 684 \mathrm{~km} \mathrm{~s}^{-1}\right)$ of CME is indicated. Taking the projection effect into account, the speeds of the flux rope and CME are comparable, validating that the flux rope serves as a driver of the CME (Cheng et al., 2013). 


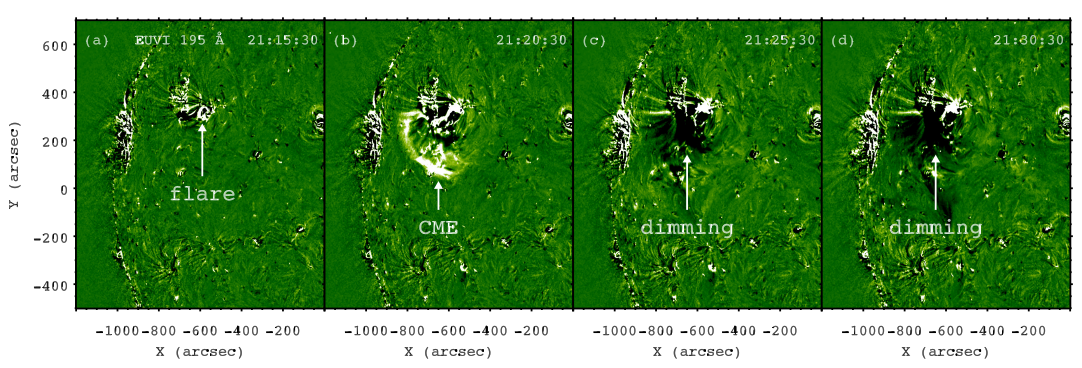

Figure 8. Base-difference images observed by STA/EUVI in $195 \AA$. The arrows point to the flare, CME front, and coronal dimming behind the CME. An animation of this figure is available in the Electronic Supplementary Material (anim195.mp4).

The prominence eruption was observed by STA/EUVI from a different viewpoint (see animation in the Electronic Supplementary Material anim195.mp4). Four $195 \AA$ base-difference images are displayed in Figure 8. In Panel a, the source region of the flare and CME is located at $\left(-590^{\prime \prime}, 300^{\prime \prime}\right)$ as indicated by the arrow. In Panel b, the arc-shaped CME leading front with enhanced intensity is pointed by the arrow. In Panels $\mathrm{c}-\mathrm{d}$, long-lasting coronal dimming behind the CME with reduced intensity and expanding area is the most striking feature (Thompson et al., 1998; Zhang, Su, and Ji, 2017). It is evident that as the CME propagates in the southeast direction, the dimming mainly extends in the south and east directions, rather than isotropically.

\subsection{Transverse Coronal-Loop Oscillations}

As mentioned before, the coronal loops to the North of the prominence are disturbed and start oscillating during the eruption (see animation in the Electronic Supplementary Material animaia.mp4). In Figure 3d1, two slices (S2 and S3) are used to investigate the transverse oscillations. The slightly curved slice S2 with a total length of $\approx 188.5^{\prime \prime}$ is parallel to the solar surface. The straight slice S3 with a length of $\approx 172^{\prime \prime}$ is vertical to the solar surface. The time-distance diagram of S2 in $171 \AA$ is displayed in Figure 9. The positions of the oscillating loop with maximal EUV intensity are denoted by the magenta-plus symbols. After the flare occurs, the coronal loop first moves southward and sways from side to side periodically. The amplitude decays with time and the oscillation lasts for three to four cycles. The initial southward movement is caused by the strong magnetic-pressure gradient after the flux rope quickly escapes the active region.

In Figure 10c, the positions of the oscillating loop along S2 are drawn with cyan circles. To precisely determine the physical parameters of the oscillation, we perform curve fitting by adopting an exponentially decaying sine function (Nisticò, Nakariakov, and Verwichte, 2013):

$$
y(t)=A_{0} \sin \left(\frac{2 \pi}{P}\left(t-t_{0}\right)+\phi_{0}\right) \mathrm{e}^{-\left(t-t_{0}\right) / \tau}+y_{0}+k\left(t-t_{0}\right)+c\left(t-t_{0}\right)^{2},
$$

where $A_{0}$ and $\phi_{0}$ stand for the initial amplitude of displacement and phase at $t_{0}$, $P$ and $\tau$ signify the period and damping time, $y_{0}$ denotes the initial loop position, 


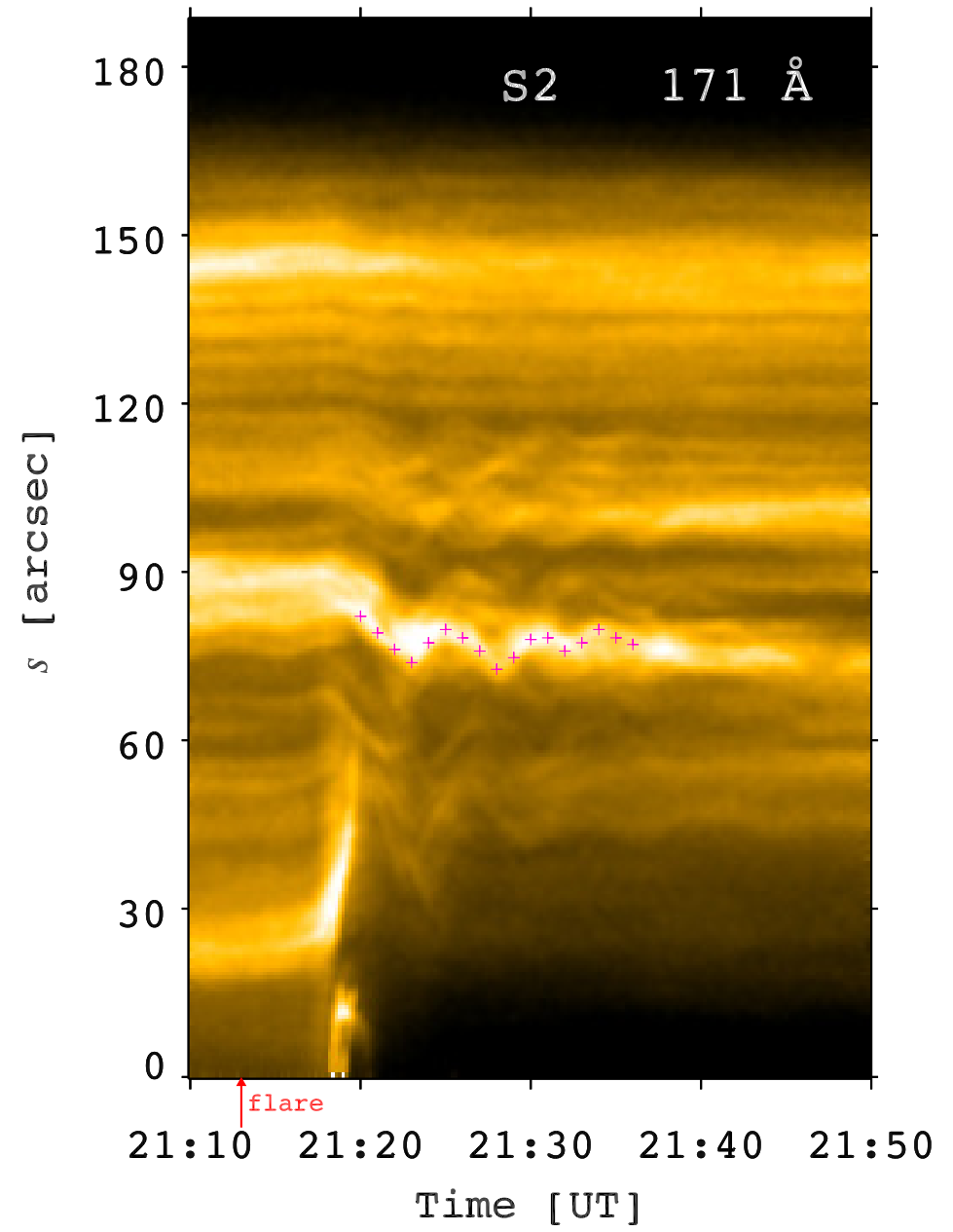

Figure 9. Time-distance diagram of S2 in $171 \AA$. The magenta-plus symbols denote the positions of the oscillating loop with maximal EUV intensity. The red arrow on the $x$-axis indicates the starting time of flare. $s=0$ and $s=188.5^{\prime \prime}$ on the $y$-axis represent the southwest and northeast endpoints of $\mathrm{S} 2$, respectively.

and $k$ and $c$ denote the coefficients of linear and quadratic terms, respectively. The results of curve fitting using mpfit.pro are drawn with a magenta-dotted line. It is evident that the transverse oscillation can be nicely described by Equation 1. In Table 2 , the fitted values of $t_{0}(\approx 21: 20: 11 \mathrm{UT}), A_{0}(\approx 3.1 \mathrm{Mm}), P(\approx 294 \mathrm{~s})$, $\tau(\approx 645 \mathrm{~s})$, and $\frac{\tau}{P}(\approx 2.2)$ are listed in the second to sixth columns. It should be emphasized that the transverse oscillation in the horizontal direction is most striking in $171 \AA$ and becomes blurred in 193 and $211 \AA$.

The time-distance diagrams of S3 in 171,193 , and $211 \AA$ are displayed in Figure 11. The positions of the loop tops are denoted by the cyan-plus symbols. Interestingly, the coronal loops start to oscillate vertically after the flare occurs. The amplitudes also decay with time and the oscillations last for three to four 


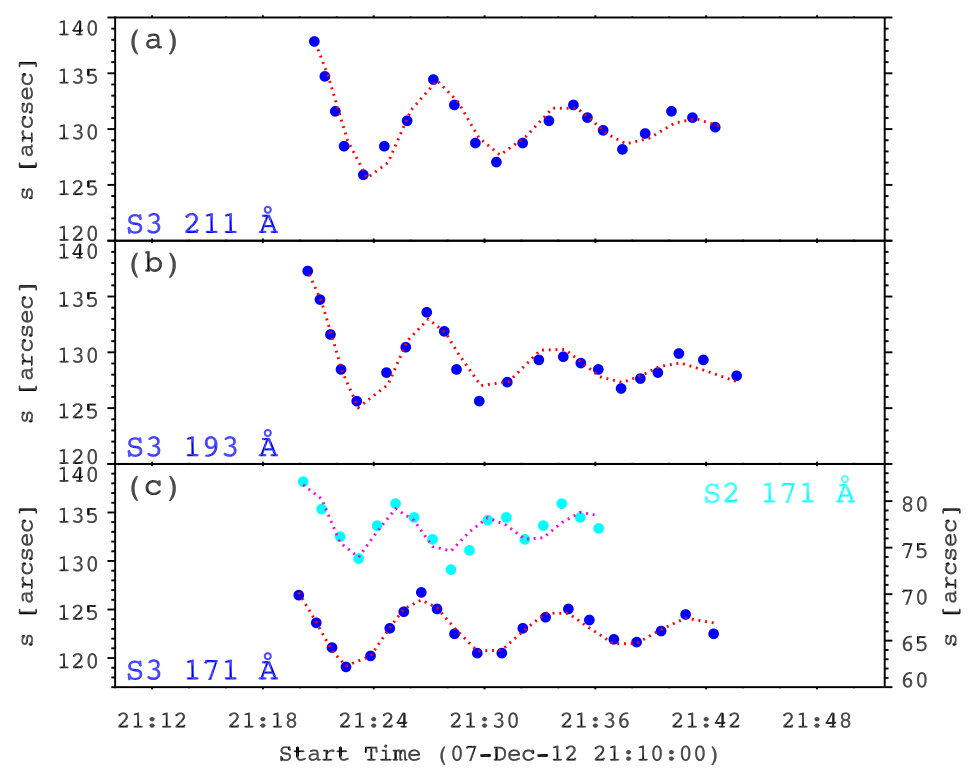

Figure 10. The positions (blue circles) of the coronal loop tops along S3 in 171, 193, and $211 \AA$. The results of curve fitting using Equation 2 are overlaid with red-dotted lines. In Panel c, the positions of the coronal loop along S2 in $171 \AA$ are plotted with cyan circles. The results of curve fitting using Equation 1 are overlaid with a magenta-dotted line.

Table 2. Parameters of the transverse coronal-loop oscillations in 171,193 , and $211 \AA . A_{0}$ is the initia amplitude at $t_{0} . P$ and $\tau$ signify the period and damping time. $h$ is the apparent height of loop top. $L$ is the loop length assuming a semi-circular shape. $C_{\mathrm{k}}$ and $C_{\mathrm{A}}$ represent the phase speed and internal Alfvén speed of the loop. $B$ denotes the magnetic field strength of the loops.

\begin{tabular}{ccccccccccc}
\hline slice & $\begin{array}{c}t_{0} \\
{[\mathrm{UT}]}\end{array}$ & $\begin{array}{c}A_{0} \\
{[\mathrm{Mm}]}\end{array}$ & $\begin{array}{c}P \\
{[\mathrm{~s}]}\end{array}$ & $\begin{array}{c}\tau \\
{[\mathrm{s}]}\end{array}$ & $\begin{array}{c}\frac{\tau}{P} \\
{[\mathrm{Mm}]}\end{array}$ & $\begin{array}{c}2 L \\
{[\mathrm{Mm}]}\end{array}$ & $\begin{array}{c}C_{\mathrm{k}} \\
{\left[\mathrm{km} \mathrm{s}^{-1}\right]}\end{array}$ & $\begin{array}{c}C_{\mathrm{A}} \\
{\left[\mathrm{km} \mathrm{s}^{-1}\right]}\end{array}$ & $\begin{array}{c}B \\
{[\mathrm{G}]}\end{array}$ \\
\hline S2_171 & $21: 20: 11$ & $3.1 \pm 0.3$ & $293.6 \pm 5$ & $645.3 \pm 39$ & 2.2 & $\ldots$ & $\ldots$ & $\ldots$ & $\ldots$ & $\ldots$ \\
S3_171 & $21: 19: 47$ & $3.4 \pm 0.3$ & $441.4 \pm 8$ & $1011.8 \pm 96$ & 2.3 & 93.5 & 587.6 & 1331.3 & 987.6 & $12-37$ \\
S3_193 & $21: 20: 18$ & $5.2 \pm 0.4$ & $407.2 \pm 5$ & $570.8 \pm 34$ & 1.4 & 100.1 & 628.6 & 1543.8 & 1145.3 & $14-43$ \\
S3_211 & $21: 20: 47$ & $5.0 \pm 0.4$ & $419.2 \pm 6$ & $641.4 \pm 43$ & 1.5 & 100.1 & 628.6 & 1499.6 & 1112.5 & $13-41$ \\
\hline
\end{tabular}

cycles. The initial inward motion indicates loop contraction or implosion before oscillation (e.g. Gosain, 2012; Sun et al., 2012b; Simões et al., 2013; Dudík et al., 2016). This is consistent with the fact that the vertical loop oscillations are caused by the eruption of the flux rope, since the magnetic pressure beneath the loops is impulsively decreased after the eruption.

The positions of the loop tops in 171,193 , and $211 \AA$ are manually determined and are drawn with blue circles in Figure 10. Likewise, we perform curve fitting by adopting an exponentially decaying sine function:

$$
y(t)=A_{0} \sin \left(\frac{2 \pi}{P}\left(t-t_{0}\right)+\phi_{0}\right) \mathrm{e}^{-\left(t-t_{0}\right) / \tau}+y_{0}+k\left(t-t_{0}\right),
$$




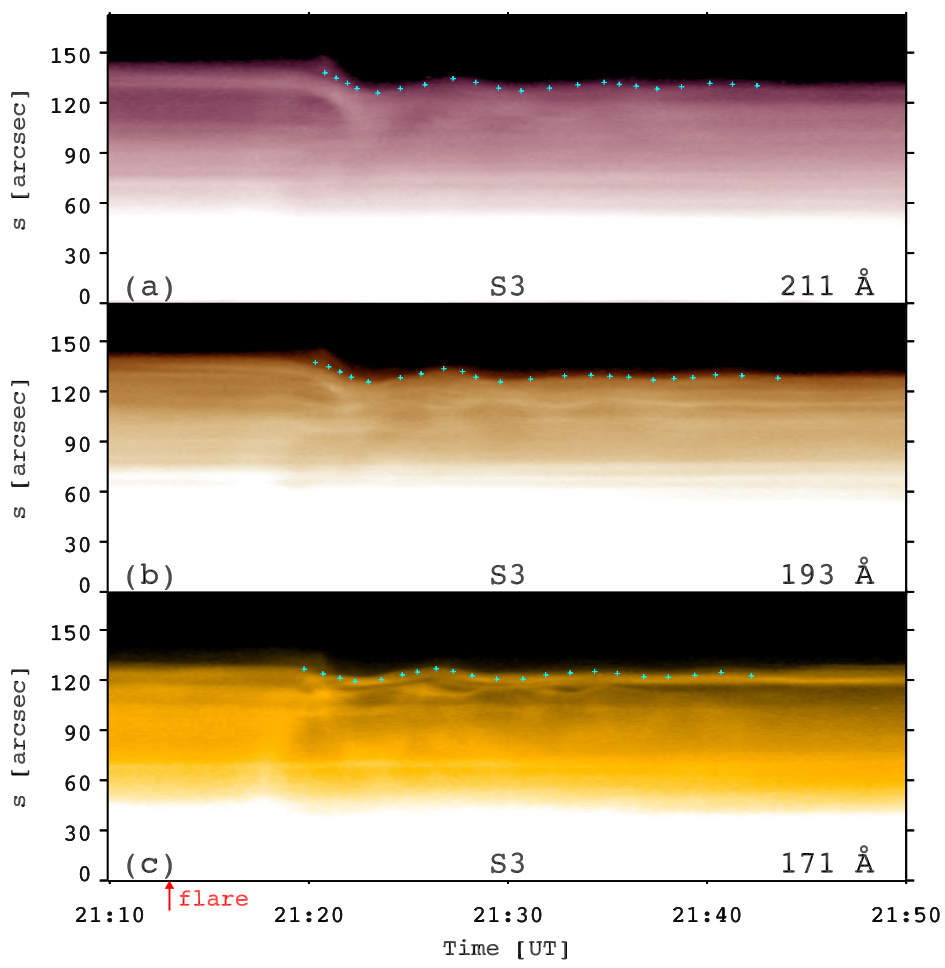

Figure 11. Time-distance diagrams of S3 in 171, 193, and $211 \AA$. The cyan-plus symbols denote the positions of the loop tops. The red arrow on the $x$-axis indicates the starting time of flare. $s=0$ and $s=172^{\prime \prime}$ on the $y$-axis represent the east and west endpoints of S3.

where the parameters have the same meanings as in Equation 1. The results of curve fitting are drawn with red-dotted lines, showing that the vertical oscillations can be well described by Equation 2. In Table 2, the fitted values of $t_{0}, A_{0}, P, \tau$, and $\frac{\tau}{P}$ are listed in the second to sixth columns. The initial amplitudes lie in the range of $3.4-5.2 \mathrm{Mm}$ with an average value of $\approx 4.5 \mathrm{Mm}$. The periods have a range of $407-441 \mathrm{~s}$ with an average value of $\approx 423 \mathrm{~s}$. The damping times are between $570 \mathrm{~s}$ and $1012 \mathrm{~s}$ with an average value of $\approx 741$ s. The corresponding quality factors $\left(\frac{\tau}{P}\right)$ are between 1.4 and 2.3 with a mean value of $\approx 1.7$, suggesting a quick attenuation (White, Verwichte, and Foullon, 2012). In $171 \AA$, the period of oscillation in the vertical direction is $\approx 1.5$ times longer than that in the horizontal direction, while the quality factors are close to each other, indicating that the transverse oscillations in both directions are independent rather than two components of the same oscillation. In Table 2, the apparent heights of the loop tops are listed in the seventh column. The loop heights in 193 and $211 \AA$ are higher than those in $171 \AA$ with lower temperature of the response function peak (Lemen et al., 2012). In other words, the coronal loops with different heights oscillate in phase (Zhang et al., 2020).

The commencements of oscillations in both horizontal and vertical directions occur during the fast rise of the flux rope (Figure $7 \mathrm{a}$ ), which is in accordance 


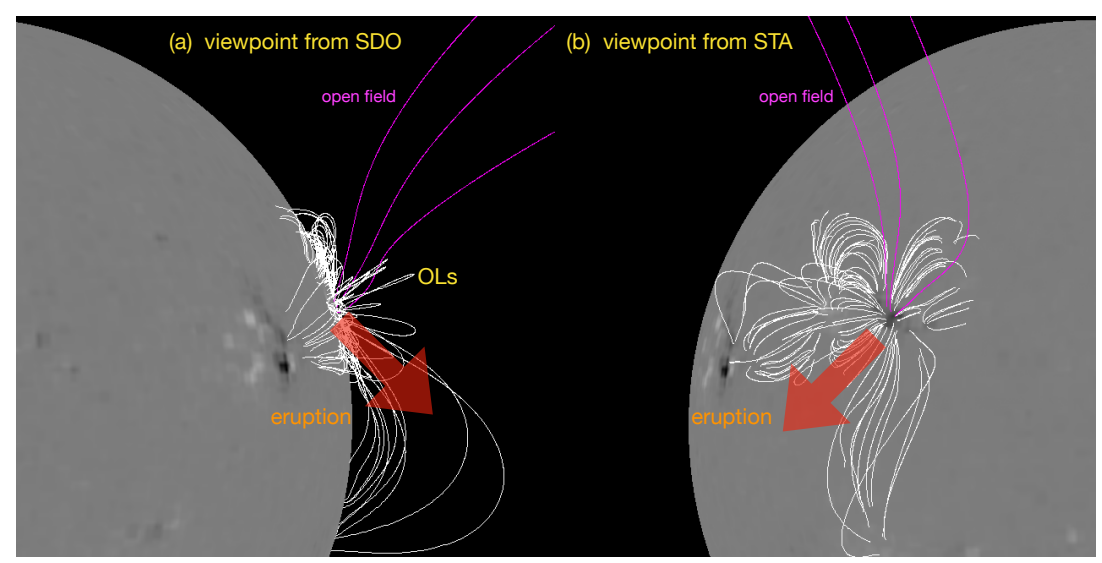

Figure 12. The 3D magnetic configuration at 18:04:00 UT from the viewpoints of SDO (a) and STA (b) using the PFSS modeling. The white and magenta lines represent the closed and open field, respectively. The red arrows indicate the directions of prominence eruption.

with the fact that the transverse oscillations are caused by the flux-rope eruption. That is to say, the coronal loops are not disturbed until the flux rope expands and propagates after a time. It is emphasized that the simultaneous oscillations in the horizontal direction and vertical directions are from different coronal loops superposed along the LOS, since the coronal loop undergoing horizontal oscillation drifts southward from the equilibrium position (see Figure 9). Moreover, the period of vertical oscillation is 1.5 times longer than the horizontal oscillation in $171 \AA$ A.

\section{Discussion}

\subsection{What is the Cause of Non-radial Eruption?}

As mentioned in Section 1, non-radial prominence eruptions are frequently observed (e.g. Williams et al., 2005; Gosain et al., 2009; Sun et al., 2012a; Bi et al., 2013; Panasenco et al., 2013; Yang et al., 2018; Mitra and Joshi, 2019; Devi et al., 2021; Guo et al., 2021b; Mancuso et al., 2021). The reported inclination angle is between $45^{\circ}$ and $90^{\circ}$. The non-radial eruption is attributed to the imbalance of magnetic pressure of the high-lying field (Aulanier et al., 2010). In the current case, the apparent inclination angle of the flux rope is $60^{\circ}$. We obtain the global 3D magnetic configuration at 18:04:00 UT using the potential-field source surface (PFSS: Schrijver and De Rosa, 2003) modeling. Figure 12 shows the configuration from the viewpoints of SDO (a) and STA (b), respectively. Combining Figure 12 and Figure 3, it is revealed that the flux rope finds a way out where the magnetic field is weaker and the escape becomes easier. This is consistent with the previous interpretation (Aulanier et al., 2010). 


\subsection{How are the Transverse Loop Oscillations Excited?}

Two decades have passed since the discovery of coronal-loop oscillations (see Li et al., 2020; Nakariakov and Kolotkov, 2020; Nakariakov et al., 2021; Wang et al., 2021, and references therein). Kink oscillation has become a topic of great interest due to its advantage in diagnosing the coronal magnetic field (Yang et al., 2020). There are several candidates in exciting kink oscillations, such as flare-induced blast waves (Nakariakov et al., 1999; Zhang, 2020), largescale coronal waves (Kumar et al., 2013), coronal jets (Dai et al., 2021), lower coronal eruptions/ejections (LCE: Zimovets and Nakariakov, 2015), coronal rains (Antolin and Verwichte, 2011), and reconnection outflows (Reeves et al., 2020). A common characteristic of the above excitation mechanisms is that the coronal loops start oscillating after being impacted. In our case, a pressure depletion is created as a result of the non-radial flux-rope eruption. Hence, the transverse loop oscillations are driven by the strong magnetic-pressure gradient of the loops (see animaia.mp4), which is rarely noticed and reported. Numerical simulations are desired to justify this mechanism.

In Figure 9 and Figure 11, there are several oscillating threads with nonzero phase differences compared to the analyzed loops. This is probably because a bundle of loops with different lengths and periods oscillate simultaneously (White and Verwichte, 2012; Nisticò, Nakariakov, and Verwichte, 2013). We focus on oscillations with clear and complete signals. It is emphasized that our analysis has LOS limitations in classifying oscillations into horizontal and vertical types based on single-point observation. The possibility of elliptically polarized transverse oscillations that are decomposed into two linearly polarized modes with different periods could not be excluded. Forward modeling and multi-point observations are required to clarify the polarization of kink oscillations (White, Verwichte, and Foullon, 2012).

\subsection{Magnetic Field Estimated from Coronal Seismology}

To estimate the magnetic field of the loops undergoing vertical oscillations, we use the observed periods and coronal seismology. The phase speed $\left[C_{\mathrm{k}}\right]$ of the standing kink oscillation is determined by the loop length $[L]$ and period (Nakariakov et al., 1999):

$$
C_{\mathrm{k}}=\frac{2 L}{P}=\sqrt{\frac{2}{1+\rho_{\mathrm{e}} / \rho_{\mathrm{i}}}} C_{\mathrm{A}},
$$

where $C_{\mathrm{A}}$ is the internal Alfvén speed, and $\rho_{\mathrm{e}}$ and $\rho_{\mathrm{i}}$ represent the external and internal plasma densities. The lengths of the oscillating loops are listed in the eighth column of Table 2 based on a semi-circular shape. The corresponding values of $C_{\mathrm{k}}$ and $C_{\mathrm{A}}$ are listed in the ninth and tenth columns, assuming that $\rho_{\mathrm{e}} / \rho_{\mathrm{i}}=0.1$ (Nakariakov and Ofman, 2001).

The magnetic-field strength of the oscillating loops is determined by $\rho_{\mathrm{i}}$ and $C_{\mathrm{A}}, B=\sqrt{4 \pi \rho_{\mathrm{i}}} C_{\mathrm{A}}$. Since the LOS depths of the oscillating loops are difficult to measure, we could not determine $\rho_{\mathrm{i}}$ precisely. Assuming that $\rho_{\mathrm{i}}$ is between 
$1.1 \times 10^{-15} \mathrm{~g} \mathrm{~cm}^{-3}$ and $1.1 \times 10^{-14} \mathrm{~g} \mathrm{~cm}^{-3}$, corresponding to the electron number density between $0.7 \times 10^{9} \mathrm{~cm}^{-3}$ and $7 \times 10^{9} \mathrm{~cm}^{-3}$ (e.g. Nakariakov and Ofman, 2001; Van Doorsselaere et al., 2008; Verwichte et al., 2009, 2013; Yuan and Van Doorsselaere, 2016; Dai et al., 2021), the magnetic-field strengths of the loops observed in different wavelengths are calculated and listed in the last column of Table 2 . Hence, the magnetic fields of the vertically oscillating loops fall in the range of $12-43 \mathrm{G}$. It is noted that the estimated magnetic field using coronal seismology has large uncertainties. On one hand, the plasma density could not be precisely determined, so that a wide range is adopted according to previous literature. On the other hand, the shape of the loops is unknown, so that a simple semi-circular shape is employed. Besides, the apparent heights $[h]$ and corresponding lengths $[L]$ of the loops are lower limits of the true values. The estimations of $C_{\mathrm{k}}, C_{\mathrm{A}}$, and $B$ in Table 2 are lower limits as well. Considering that the loops undergoing horizontal and vertical oscillations are heavily superposed along the LOS, the height of oscillating loop in the horizontal direction is hard to determine. Therefore, the horizontal oscillation is not used for seismology.

\section{Summary}

In this article, we investigate the transverse coronal loop oscillations induced by the eruption of a prominence-carrying flux rope on 7 December 2012. The main results are as follows:

1. The flux rope originating from AR 11621 is observed in various EUV wavelengths, suggesting its multi-thermal nature. The early evolution of the flux rope is divided into two phases: a slow rise phase at a speed of $\approx 230 \mathrm{~km} \mathrm{~s}^{-1}$ and a fast rise phase at a speed of $\approx 706 \mathrm{~km} \mathrm{~s}^{-1}$. The eruption generates a C5.8 flare and the onset of fast rise is consistent with the HXR peak time of the flare. The embedded prominence has a lower speed of $\approx 452 \mathrm{~km} \mathrm{~s}^{-1}$. The propagation of the flux rope is along the southwest direction in the FOV of AIA. Hence, the inclination angle between the direction of flux rope eruption and the local solar normal reaches $\approx 60^{\circ}$, suggesting a typical non-radial eruption.

2. During the early eruption of the flux rope, the nearby coronal loops are disturbed and experience kink-mode oscillations. The oscillation in the horizontal direction has an initial amplitude of $\approx 3.1 \mathrm{Mm}$, a period of $\approx 294 \mathrm{~s}$, and a damping time of $\approx 645 \mathrm{~s}$. It is most striking in $171 \AA$ and lasts for three to four cycles. The oscillations in the vertical directions are observed mainly in 171,193 , and $211 \AA$. The initial amplitudes lie in the range of $3.4-5.2 \mathrm{Mm}$, with an average value of $4.5 \mathrm{Mm}$. The periods are between 407 seconds and 441 seconds, with an average value of 423 seconds ( $\approx 7$ minutes). The oscillations are damping and last for nearly four cycles. The damping times lie in the range of $570-1012$ seconds, with an average value of 741 seconds.

3. Assuming a semi-circular shape of the vertically oscillating loops, we calculate the loop lengths according to their heights. Using the observed periods, we carry out coronal seismology and estimate the internal Alfvén speeds $\left(988-1145 \mathrm{~km} \mathrm{~s}^{-1}\right)$ and the magnetic-field strengths $(12-43 \mathrm{G})$ of the oscillating loops. 
Acknowledgments The authors thank the reviewer for valuable comments and suggestions. We thank L. Feng and Y.N. Su in Purple Mountain Observatory for helpful discussions. SDO is a mission of NASA's Living With a Star Program. AIA and HMI data are courtesy of the NASA/SDO science teams. This work is funded by the National Key R\&D Program of China 2021YFA1600502 (2021YFA1600500), NSFC grants (No. 11790302, 11773079, 11973012, 11973092, 12073081), the International Cooperation and Interchange Program (11961131002), CAS Key Laboratory of Solar Activity, National Astronomical Observatories (KLSA202113), the Strategic Priority Research Program on Space Science, CAS (XDA15052200, XDA15320301) and the mobility program (M-0068) of the Sino-German Science Center.

\section{Declarations}

Disclosure of Potential Conflicts of Interest The authors declare that they have no conflicts of interest.

\section{References}

Amari, T., Canou, A., Aly, J.-J.: 2014, Characterizing and predicting the magnetic environment leading to solar eruptions. Nature 514, 465. DOI. ADS.

Antolin, P., Verwichte, E.: 2011, Transverse Oscillations of Loops with Coronal Rain Observed by Hinode/Solar Optical Telescope. Astrophys. J. 736, 121. DOI. ADS.

Antolin, P., De Moortel, I., Van Doorsselaere, T., Yokoyama, T.: 2017, Observational Signatures of Transverse Magnetohydrodynamic Waves and Associated Dynamic Instabilities in Coronal Flux Tubes. Astrophys. J. 836, 219. DOI. ADS.

Aschwanden, M.J., Fletcher, L., Schrijver, C.J., Alexander, D.: 1999, Coronal Loop Oscillations Observed with the Transition Region and Coronal Explorer. Astrophys. J. 520, 880. DOI. ADS.

Aschwanden, M.J., de Pontieu, B., Schrijver, C.J., Title, A.M.: 2002, Transverse Oscillations in Coronal Loops Observed with TRACE II. Measurements of Geometric and Physical Parameters. Solar Phys. 206, 99. DOI. ADS.

Aulanier, G., Török, T., Démoulin, P., DeLuca, E.E.: 2010, Formation of Torus-Unstable Flux Ropes and Electric Currents in Erupting Sigmoids. Astrophys. J. 708, 314. DOI. ADS.

Bi, Y., Jiang, Y., Yang, J., Zheng, R., Hong, J., Li, H., Yang, D., Yang, B.: 2013, Analysis of the Simultaneous Rotation and Non-radial Propagation of an Eruptive Filament. Astrophys. J. 773, 162. DOI. ADS.

Brown, J.C.: 1971, The Deduction of Energy Spectra of Non-Thermal Electrons in Flares from the Observed Dynamic Spectra of Hard X-Ray Bursts. Solar Phys. 18, 489. DOI. ADS.

Brueckner, G.E., Howard, R.A., Koomen, M.J., Korendyke, C.M., Michels, D.J., Moses, J.D., Socker, D.G., Dere, K.P., Lamy, P.L., Llebaria, A., Bout, M.V., Schwenn, R., Simnett, G.M., Bedford, D.K., Eyles, C.J.: 1995, The Large Angle Spectroscopic Coronagraph (LASCO). Solar Phys. 162, 357. DOI. ADS.

Canou, A., Amari, T., Bommier, V., Schmieder, B., Aulanier, G., Li, H.: 2009, Evidence for a Pre-Eruptive Twisted Flux Rope Using the Themis Vector Magnetograph. Astrophys. J. Lett. 693, L27. DOI. ADS.

Chen, F., Peter, H.: 2015, Using coronal seismology to estimate the magnetic field strength in a realistic coronal model. Astron. Astrophys. 581, A137. DOI. ADS.

Chen, H., Yang, J., Ji, K., Duan, Y.: 2019, Observational Analysis on the Early Evolution of a CME Flux Rope: Preflare Reconnection and Flux Rope's Footpoint Drift. Astrophys. J. 887, 118. DOI. ADS.

Chen, J.: 2017, Physics of erupting solar flux ropes: Coronal mass ejections (CMEs)—Recent advances in theory and observation. Physics of Plasmas 24, 090501. DOI. ADS.

Cheng, X., Guo, Y., Ding, M.: 2017, Origin and Structures of Solar Eruptions I: Magnetic Flux Rope. Science China Earth Sciences 60, 1383. DOI. ADS. 
Cheng, X., Zhang, J., Liu, Y., Ding, M.D.: 2011, Observing Flux Rope Formation During the Impulsive Phase of a Solar Eruption. Astrophys. J. Lett. 732, L25. DOI. ADS.

Cheng, X., Zhang, J., Saar, S.H., Ding, M.D.: 2012, Differential Emission Measure Analysis of Multiple Structural Components of Coronal Mass Ejections in the Inner Corona. Astrophys. J. 761, 62. DOI. ADS.

Cheng, X., Zhang, J., Ding, M.D., Liu, Y., Poomvises, W.: 2013, The Driver of Coronal Mass Ejections in the Low Corona: A Flux Rope. Astrophys. J. 763, 43. DOI. ADS.

Cheng, X., Ding, M.D., Zhang, J., Srivastava, A.K., Guo, Y., Chen, P.F., Sun, J.Q.: 2014, On the Relationship Between a Hot-channel-like Solar Magnetic Flux Rope and its Embedded Prominence. Astrophys. J. Lett. 789, L35. DOI. ADS.

Dai, J., Zhang, Q.M., Su, Y.N., Ji, H.S.: 2021, Transverse oscillation of a coronal loop induced by a flare-related jet. Astron. Astrophys. 646, A12. DOI. ADS.

Dere, K.P., Brueckner, G.E., Howard, R.A., Michels, D.J., Delaboudinière, J.P.: 1999, LASCO and EIT Observations of Helical Structure in Coronal Mass Ejections. Astrophys. J. 516, 465. DOI. ADS.

Devi, P., Démoulin, P., Chandra, R., Joshi, R., Schmieder, B., Joshi, B.: 2021, Observations of a prominence eruption and loop contraction. Astron. Astrophys. 647, A85. DOI. ADS.

Dudík, J., Polito, V., Janvier, M., Mulay, S.M., Karlický, M., Aulanier, G., Del Zanna, G., Dzifčáková, E., Mason, H.E., Schmieder, B.: 2016, Slipping Magnetic Reconnection, Chromospheric Evaporation, Implosion, and Precursors in the 2014 September 10 X1.6-Class Solar Flare. Astrophys. J. 823, 41. DOI. ADS.

Goddard, C.R., Nakariakov, V.M.: 2016, Dependence of kink oscillation damping on the amplitude. Astron. Astrophys. 590, L5. DOI. ADS.

Goddard, C.R., Nisticò, G., Nakariakov, V.M., Zimovets, I.V.: 2016, A statistical study of decaying kink oscillations detected using SDO/AIA. Astron. Astrophys. 585, A137. DOI. ADS.

Goossens, M., Andries, J., Aschwanden, M.J.: 2002, Coronal loop oscillations. An interpretation in terms of resonant absorption of quasi-mode kink oscillations. Astron. Astrophys. 394, L39. DOI. ADS.

Gosain, S.: 2012, Evidence for Collapsing Fields in the Corona and Photosphere during the 2011 February 15 X2.2 Flare: SDO/AIA and HMI Observations. Astrophys. J. 749, 85. DOI. ADS.

Gosain, S., Schmieder, B., Venkatakrishnan, P., Chandra, R., Artzner, G.: 2009, 3D Evolution of a Filament Disappearance Event Observed by STEREO. Solar Phys. 259, 13. DOI. ADS.

Gou, T., Liu, R., Kliem, B., Wang, Y., Veronig, A.M.: 2019, The Birth of A Coronal Mass Ejection. Sci. Adv. 5, 7004. DOI. ADS.

Green, L.M., Kliem, B.: 2009, Flux Rope Formation Preceding Coronal Mass Ejection Onset. Astrophys. J. Lett. 700, L83. DOI. ADS.

Green, L.M., Kliem, B., Wallace, A.J.: 2011, Photospheric flux cancellation and associated flux rope formation and eruption. Astron. Astrophys. 526, A2. DOI. ADS.

Guo, J.H., Ni, Y.W., Qiu, Y., Zhong, Z., Guo, Y., Chen, P.F.: 2021a, Magnetic Twists of Solar Filaments. Astrophys. J. 917, 81. DOI. ADS.

Guo, Y., Zhong, Z., Ding, M.D., Chen, P.F., Xia, C., Keppens, R.: 2021b, Data-constrained Magnetohydrodynamic Simulation of a Long-duration Eruptive Flare. Astrophys. J. 919, 39. DOI. ADS.

Hannah, I.G., Kontar, E.P.: 2013, Multi-thermal dynamics and energetics of a coronal mass ejection in the low solar atmosphere. Astron. Astrophys. 553, A10. DOI. ADS.

He, W., Jiang, C., Zou, P., Duan, A., Feng, X., Zuo, P., Wang, Y.: 2020, Data-driven MHD Simulation of the Formation and Initiation of a Large-scale Preflare Magnetic Flux Rope in AR 12371. Astrophys. J. 892, 9. DOI. ADS.

Howard, R.A., Moses, J.D., Vourlidas, A., Newmark, J.S., Socker, D.G., Plunkett, S.P., Korendyke, C.M., Cook, J.W., Hurley, A., Davila, J.M., Thompson, W.T., St Cyr, O.C., Mentzell, E., Mehalick, K., Lemen, J.R., Wuelser, J.P., Duncan, D.W., Tarbell, T.D., Wolfson, C.J., Moore, A., Harrison, R.A., Waltham, N.R., Lang, J., Davis, C.J., Eyles, C.J., Mapson-Menard, H., Simnett, G.M., Halain, J.P., Defise, J.M., Mazy, E., Rochus, P., Mercier, R., Ravet, M.F., Delmotte, F., Auchère, F., Delaboudinière, J.P., Bothmer, V., Deutsch, W., Wang, D., Rich, N., Cooper, S., Stephens, V., Maahs, G., Baugh, R., McMullin, D., Carter, T.: 2008, Sun Earth Connection Coronal and Heliospheric Investigation (SECCHI). Space Sci. Rev. 136, 67. DOI. ADS. 
Inoue, S., Shiota, D., Bamba, Y., Park, S.-H.: 2018, Magnetohydrodynamic Modeling of a Solar Eruption Associated with an X9.3 Flare Observed in the Active Region 12673. Astrophys. J. 867, 83. DOI. ADS.

James, A.W., Valori, G., Green, L.M., Liu, Y., Cheung, M.C.M., Guo, Y., van Driel-Gesztelyi, L.: 2018, An Observationally Constrained Model of a Flux Rope that Formed in the Solar Corona. Astrophys. J. Lett. 855, L16. DOI. ADS.

Janvier, M., Aulanier, G., Démoulin, P.: 2015, From Coronal Observations to MHD Simulations, the Building Blocks for 3D Models of Solar Flares (Invited Review). Solar Phys. 290, 3425. DOI. ADS.

Jiang, C., Zou, P., Feng, X., Hu, Q., Liu, R., Vemareddy, P., Duan, A., Zuo, P., Wang, Y., Wei, F.: 2018, Magnetohydrodynamic Simulation of the X9.3 Flare on 2017 September 6: Evolving Magnetic Topology. Astrophys. J. 869, 13. DOI. ADS.

Joshi, N.C., Magara, T., Inoue, S.: 2014, Formation of a Compound Flux Rope by the Merging of Two Filament Channels, the Associated Dynamics, and its Stability. Astrophys. J. 795, 4. DOI. ADS

Kaiser, M.L., Kucera, T.A., Davila, J.M., St. Cyr, O.C., Guhathakurta, M., Christian, E.: 2008, The STEREO Mission: An Introduction. Space Sci. Rev. 136, 5. DOI. ADS.

Kim, S., Nakariakov, V.M., Cho, K.-S.: 2014, Vertical Kink Oscillation of a Magnetic Flux Rope Structure in the Solar Corona. Astrophys. J. Lett. 797, L22. DOI. ADS.

Kliem, B., Su, Y.N., van Ballegooijen, A.A., DeLuca, E.E.: 2013, Magnetohydrodynamic Modeling of the Solar Eruption on 2010 April 8. Astrophys. J. 779, 129. DOI. ADS.

Kumar, P., Cho, K.-S., Chen, P.F., Bong, S.-C., Park, S.-H.: 2013, Multiwavelength Study of a Solar Eruption from AR NOAA 11112: II. Large-Scale Coronal Wave and Loop Oscillation. Solar Phys. 282, 523. DOI. ADS.

Lemen, J.R., Title, A.M., Akin, D.J., Boerner, P.F., Chou, C., Drake, J.F., Duncan, D.W., Edwards, C.G., Friedlaender, F.M., Heyman, G.F., Hurlburt, N.E., Katz, N.L., Kushner, G.D., Levay, M., Lindgren, R.W., Mathur, D.P., McFeaters, E.L., Mitchell, S., Rehse, R.A., Schrijver, C.J., Springer, L.A., Stern, R.A., Tarbell, T.D., Wuelser, J.-P., Wolfson, C.J., Yanari, C., Bookbinder, J.A., Cheimets, P.N., Caldwell, D., Deluca, E.E., Gates, R., Golub, L., Park, S., Podgorski, W.A., Bush, R.I., Scherrer, P.H., Gummin, M.A., Smith, P., Auker, G., Jerram, P., Pool, P., Soufli, R., Windt, D.L., Beardsley, S., Clapp, M., Lang, J., Waltham, N.: 2012, The Atmospheric Imaging Assembly (AIA) on the Solar Dynamics Observatory (SDO). Solar Phys. 275, 17. DOI. ADS.

Li, B., Antolin, P., Guo, M.-Z., Kuznetsov, A.A., Pascoe, D.J., Van Doorsselaere, T., Vasheghani Farahani, S.: 2020, Magnetohydrodynamic Fast Sausage Waves in the Solar Corona. Space Sci. Rev. 216, 136. DOI. ADS.

Li, D., Ning, Z.J., Huang, Y., Chen, N.-H., Zhang, Q.M., Su, Y.N., Su, W.: 2017, Doppler Shift Oscillations from a Hot Line Observed by IRIS. Astrophys. J. 849, 113. DOI. ADS.

Li, D., Yuan, D., Su, Y.N., Zhang, Q.M., Su, W., Ning, Z.J.: 2018, Non-damping oscillations at flaring loops. Astron. Astrophys. 617, A86. DOI. ADS.

Liu, R.: 2020, Magnetic flux ropes in the solar corona: structure and evolution toward eruption. Res. Astron. Astrophys. 20, 165. DOI. ADS.

Liu, R., Kliem, B., Titov, V.S., Chen, J., Wang, Y., Wang, H., Liu, C., Xu, Y., Wiegelmann, T.: 2016, Structure, Stability, and Evolution of Magnetic Flux Ropes from the Perspective of Magnetic Twist. Astrophys. J. 818, 148. DOI. ADS.

Mancuso, S., Bemporad, A., Frassati, F., Barghini, D., Giordano, S., Telloni, D., Taricco, C.: 2021, Radio evidence for a shock wave reflected by a coronal hole. Astron. Astrophys. 651, L14. DOI. ADS.

McCauley, P.I., Su, Y.N., Schanche, N., Evans, K.E., Su, C., McKillop, S., Reeves, K.K.: 2015, Prominence and Filament Eruptions Observed by the Solar Dynamics Observatory: Statistical Properties, Kinematics, and Online Catalog. Solar Phys. 290, 1703. DOI. ADS.

Meegan, C., Lichti, G., Bhat, P.N., Bissaldi, E., Briggs, M.S., Connaughton, V., Diehl, R., Fishman, G., Greiner, J., Hoover, A.S., van der Horst, A.J., von Kienlin, A., Kippen, R.M., Kouveliotou, C., McBreen, S., Paciesas, W.S., Preece, R., Steinle, H., Wallace, M.S., Wilson, R.B., Wilson-Hodge, C.: 2009, The Fermi Gamma-ray Burst Monitor. Astrophys. J. 702, 791. DOI. ADS.

Mitra, P.K., Joshi, B.: 2019, Preflare Processes, Flux Rope Activation, Large-scale Eruption, and Associated X-class Flare from the Active Region NOAA 11875. Astrophys. J. 884, 46. DOI. ADS.

Nakariakov, V.M., Kolotkov, D.Y.: 2020, Magnetohydrodynamic Waves in the Solar Corona. Annu. Rev. Astron. Astr. 58, 441. DOI. ADS. 
Nakariakov, V.M., Ofman, L.: 2001, Determination of the coronal magnetic field by coronal loop oscillations. Astron. Astrophys. 372, L53. DOI. ADS.

Nakariakov, V.M., Ofman, L., Deluca, E.E., Roberts, B., Davila, J.M.: 1999, TRACE observation of damped coronal loop oscillations: Implications for coronal heating. Science 285, 862. DOI. ADS.

Nakariakov, V.M., Anfinogentov, S.A., Antolin, P., Jain, R., Kolotkov, D.Y., Kupriyanova, E.G., Li, D., Magyar, N., Nisticò, G., Pascoe, D.J., Srivastava, A.K., Terradas, J., Vasheghani Farahani, S., Verth, G., Yuan, D., Zimovets, I.V.: 2021, Kink Oscillations of Coronal Loops. Space Sci. Rev. 217, 73. DOI. ADS.

Nechaeva, A., Zimovets, I.V., Nakariakov, V.M., Goddard, C.R.: 2019, Catalog of Decaying Kink Oscillations of Coronal Loops in the 24th Solar Cycle. Astrophys. J. Suppl. S. 241, 31. DOI. ADS.

Nindos, A., Patsourakos, S., Vourlidas, A., Tagikas, C.: 2015, How Common Are Hot Magnetic Flux Ropes in the Low Solar Corona? A Statistical Study of EUV Observations. Astrophys. J. 808, 117. DOI. ADS.

Nindos, A., Patsourakos, S., Vourlidas, A., Cheng, X., Zhang, J.: 2020, When do solar erupting hot magnetic flux ropes form? Astron. Astrophys. 642, A109. DOI. ADS.

Nisticò, G., Nakariakov, V.M., Verwichte, E.: 2013, Decaying and decayless transverse oscillations of a coronal loop. Astron. Astrophys. 552, A57. DOI. ADS.

Ofman, L., Aschwanden, M.J.: 2002, Damping Time Scaling of Coronal Loop Oscillations Deduced from Transition Region and Coronal Explorer Observations. Astrophys. J. Lett. 576, L153. DOI. ADS.

Panasenco, O., Martin, S.F., Velli, M., Vourlidas, A.: 2013, Origins of Rolling, Twisting, and Non-radial Propagation of Eruptive Solar Events. Solar Phys. 287, 391. DOI. ADS.

Patsourakos, S., Vourlidas, A., Török, T., Kliem, B., Antiochos, S.K., Archontis, V., Aulanier, G., Cheng, X., Chintzoglou, G., Georgoulis, M.K., Green, L.M., Leake, J.E., Moore, R., Nindos, A., Syntelis, P., Yardley, S.L., Yurchyshyn, V., Zhang, J.: 2020, Decoding the PreEruptive Magnetic Field Configurations of Coronal Mass Ejections. Space Sci. Rev. 216, 131. DOI. ADS.

Reeves, K.K., Polito, V., Chen, B., Galan, G., Yu, S., Liu, W., Li, G.: 2020, Hot Plasma Flows and Oscillations in the Loop-top Region During the 2017 September 10 X8.2 Solar Flare. Astrophys. J. 905, 165. DOI. ADS.

Régnier, S., Walsh, R.W., Alexander, C.E.: 2011, A new look at a polar crown cavity as observed by SDO/AIA. Structure and dynamics. Astron. Astrophys. 533, L1. DOI. ADS.

Ruderman, M.S., Roberts, B.: 2002, The Damping of Coronal Loop Oscillations. Astrophys. J. 577, 475. DOI. ADS.

Rust, D.M., Kumar, A.: 1996, Evidence for Helically Kinked Magnetic Flux Ropes in Solar Eruptions. Astrophys. J. Lett. 464, L199. DOI. ADS.

Savcheva, A.S., Green, L.M., van Ballegooijen, A.A., DeLuca, E.E.: 2012, Photospheric Flux Cancellation and the Build-up of Sigmoidal Flux Ropes on the Sun. Astrophys. J. 759, 105. DOI. ADS.

Scherrer, P.H., Schou, J., Bush, R.I., Kosovichev, A.G., Bogart, R.S., Hoeksema, J.T., Liu, Y., Duvall, T.L., Zhao, J., Title, A.M., Schrijver, C.J., Tarbell, T.D., Tomczyk, S.: 2012, The Helioseismic and Magnetic Imager (HMI) Investigation for the Solar Dynamics Observatory (SDO). Solar Phys. 275, 207. DOI. ADS.

Schrijver, C.J., De Rosa, M.L.: 2003, Photospheric and heliospheric magnetic fields. Solar Phys. 212, 165. DOI. ADS.

Simões, P.J.A., Fletcher, L., Hudson, H.S., Russell, A.J.B.: 2013, Implosion of Coronal Loops during the Impulsive Phase of a Solar Flare. Astrophys. J. 777, 152. DOI. ADS.

Srivastava, A.K., Goossens, M.: 2013, X6.9-class Flare-induced Vertical Kink Oscillations in a Large-scale Plasma Curtain as Observed by the Solar Dynamics Observatory/Atmospheric Imaging Assembly. Astrophys. J. 777, 17. DOI. ADS.

Sun, X., Hoeksema, J.T., Liu, Y., Chen, Q., Hayashi, K.: 2012a, A Non-radial Eruption in a Quadrupolar Magnetic Configuration with a Coronal Null. Astrophys. J. 757, 149. DOI. ADS.

Sun, X., Hoeksema, J.T., Liu, Y., Wiegelmann, T., Hayashi, K., Chen, Q., Thalmann, J.: 2012b, Evolution of Magnetic Field and Energy in a Major Eruptive Active Region Based on SDO/HMI Observation. Astrophys. J. 748, 77. DOI. ADS.

Terradas, J., Oliver, R., Ballester, J.L.: 2006, Damping of Kink Oscillations in Curved Coronal Loops. Astrophys. J. Lett. 650, L91. DOI. ADS. 
Thompson, B.J., Plunkett, S.P., Gurman, J.B., Newmark, J.S., St. Cyr, O.C., Michels, D.J.: 1998, SOHO/EIT observations of an Earth-directed coronal mass ejection on May 12, 1997. Geophys. Res. Lett. 25, 2465. DOI. ADS.

Titov, V.S., Démoulin, P.: 1999, Basic topology of twisted magnetic configurations in solar flares. Astron. Astrophys. 351, 707. ADS.

Van Doorsselaere, T., Nakariakov, V.M., Young, P.R., Verwichte, E.: 2008, Coronal magnetic field measurement using loop oscillations observed by Hinode/EIS. Astron. Astrophys. 487, L17. DOI. ADS.

Verwichte, E., Kohutova, P.: 2017, Excitation and evolution of vertically polarised transverse loop oscillations by coronal rain. Astron. Astrophys. 601, L2. DOI. ADS.

Verwichte, E., Aschwanden, M.J., Van Doorsselaere, T., Foullon, C., Nakariakov, V.M.: 2009, Seismology of a Large Solar Coronal Loop from EUVI/STEREO Observations of its Transverse Oscillation. Astrophys. J. 698, 397. DOI. ADS.

Verwichte, E., Van Doorsselaere, T., White, R.S., Antolin, P.: 2013, Statistical seismology of transverse waves in the solar corona. Astron. Astrophys. 552, A138. DOI. ADS.

Vourlidas, A., Lynch, B.J., Howard, R.A., Li, Y.: 2013, How Many CMEs Have Flux Ropes? Deciphering the Signatures of Shocks, Flux Ropes, and Prominences in Coronagraph Observations of CMEs. Solar Phys. 284, 179. DOI. ADS.

Wang, H., Cao, W., Liu, C., Xu, Y., Liu, R., Zeng, Z., Chae, J., Ji, H.: 2015, Witnessing magnetic twist with high-resolution observation from the 1.6-m New Solar Telescope. Nat. Comm. 6, 7008. DOI. ADS.

Wang, T.J., Solanki, S.K.: 2004, Vertical oscillations of a coronal loop observed by TRACE. Astron. Astrophys. 421, L33. DOI. ADS.

Wang, T., Ofman, L., Yuan, D., Reale, F., Kolotkov, D.Y., Srivastava, A.K.: 2021, Slow-Mode Magnetoacoustic Waves in Coronal Loops. Space Sci. Rev. 217, 34. DOI. ADS.

White, R.S., Verwichte, E.: 2012, Transverse coronal loop oscillations seen in unprecedented detail by AIA/SDO. Astron. Astrophys. 537, A49. DOI. ADS.

White, R.S., Verwichte, E., Foullon, C.: 2012, First observation of a transverse vertical oscillation during the formation of a hot post-flare loop. Astron. Astrophys. 545, A129. DOI. ADS.

Williams, D.R., Török, T., Démoulin, P., van Driel-Gesztelyi, L., Kliem, B.: 2005, Eruption of a Kink-unstable Filament in NOAA Active Region 10696. Astrophys. J. Lett. 628, L163. DOI. ADS

Xia, C., Keppens, R., Antolin, P., Porth, O.: 2014, Simulating the in Situ Condensation Process of Solar Prominences. Astrophys. J. Lett. 792, L38. DOI. ADS.

Xue, Z., Yan, X., Yang, L., Wang, J., Zhao, L.: 2017, Observing Formation of Flux Rope by Tether-cutting Reconnection in the Sun. Astrophys. J. Lett. 840, L23. DOI. ADS.

Yan, X.L., Yang, L.H., Xue, Z.K., Mei, Z.X., Kong, D.F., Wang, J.C., Li, Q.L.: 2018, Simultaneous Observation of a Flux Rope Eruption and Magnetic Reconnection during an X-class Solar Flare. Astrophys. J. Lett. 853, L18. DOI. ADS.

Yang, J., Dai, J., Chen, H., Li, H., Jiang, Y.: 2018, Filament Eruption with a Deflection of Nearly 90 Degrees. Astrophys. J. 862, 86. DOI. ADS.

Yang, Z., Bethge, C., Tian, H., Tomczyk, S., Morton, R., Del Zanna, G., McIntosh, S.W., Karak, B.B., Gibson, S., Samanta, T., He, J., Chen, Y., Wang, L.: 2020, Global maps of the magnetic field in the solar corona. Science 369, 694. DOI. ADS.

Yuan, D., Van Doorsselaere, T.: 2016, Forward Modeling of Standing Kink Modes in Coronal Loops. II. Applications. Astrophys. J. Suppl. S. 223, 24. DOI. ADS.

Zhang, J., Cheng, X., Ding, M.-D.: 2012, Observation of an evolving magnetic flux rope before and during a solar eruption. Nat. Comm. 3, 747. DOI. ADS.

Zhang, Q.M.: 2020, Simultaneous transverse oscillations of a coronal loop and a filament excited by a circular-ribbon flare. Astron. Astrophys. 642, A159. DOI. ADS.

Zhang, Q.M.: 2021, A revised cone model and its application to non-radial prominence eruptions. Astron. Astrophys. 653, L2. DOI. ADS.

Zhang, Q.M., Su, Y.N., Ji, H.S.: 2017, Pre-flare coronal dimmings. Astron. Astrophys. 598, A3. DOI. ADS.

Zhang, Q.M., Ning, Z.J., Guo, Y., Zhou, T.H., Cheng, X., Ji, H.S., Feng, L., Wiegelmann, T.: 2015, Multiwavelength Observations of a Partially Eruptive Filament on 2011 September 8. Astrophys. J. 805, 4. DOI. ADS.

Zhang, Q.M., Dai, J., Xu, Z., Li, D., Lu, L., Tam, K.V., Xu, A.A.: 2020, Transverse coronal loop oscillations excited by homologous circular-ribbon flares. Astron. Astrophys. 638, A32. DOI. ADS. 
Zhou, Z., Zhang, J., Wang, Y., Liu, R., Chintzoglou, G.: 2017, Toward Understanding the 3D Structure and Evolution of Magnetic Flux Ropes in an Extremely Long Duration Eruptive Flare. Astrophys. J. 851, 133. DOI. ADS.

Zimovets, I.V., Nakariakov, V.M.: 2015, Excitation of kink oscillations of coronal loops: statistical study. Astron. Astrophys. 577, A4. DOI. ADS.

Zimovets, I.V., McLaughlin, J.A., Srivastava, A.K., Kolotkov, D.Y., Kuznetsov, A.A., Kupriyanova, E.G., Cho, I.-H., Inglis, A.R., Reale, F., Pascoe, D.J., Tian, H., Yuan, D., Li, D., Zhang, Q.M.: 2021, Quasi-Periodic Pulsations in Solar and Stellar Flares: A Review of Underpinning Physical Mechanisms and Their Predicted Observational Signatures. Space Sci. Rev. 217, 66. DOI. ADS. 